\title{
Prey and Non-prey Arthropods Sharing a Host Plant: Effects on Induced Volatile Emission and Predator Attraction
}

\author{
Jetske G. de Boer • Cornelis A. Hordijk • \\ Maarten A. Posthumus • Marcel Dicke
}

Received: 5 June 2007 /Revised: 1 November 2007 / Accepted: 14 November 2007 / Published online: 10 January 2008

(C) The Author(s) 2007

\begin{abstract}
It is well established that plants infested with a single herbivore species can attract specific natural enemies through the emission of herbivore-induced volatiles. However, it is less clear what happens when plants are simultaneously attacked by more than one species. We analyzed volatile emissions of lima bean and cucumber plants upon multi-species herbivory by spider mites (Tetranychus urticae) and caterpillars (Spodoptera exigua) in comparison to single-species herbivory. Upon herbivory by single or multiple species, lima bean and cucumber plants emitted volatile blends that comprised mostly the same compounds. To detect additive, synergistic, or antagonistic effects, we compared the multi-species herbivory volatile blend with the sum of the volatile blends induced by each of the herbivore species feeding alone. In lima bean, the majority of
\end{abstract}

J. G. de Boer $(\bowtie) \cdot$ M. Dicke

Laboratory of Entomology, Wageningen University,

P.O. Box 8031, $6700 \mathrm{EH}$

Wageningen, The Netherlands

e-mail: j.g.de.boer@rug.nl

URL: www.insect-wur.nl

C. A. Hordijk

Netherlands Institute for Ecology, Centre for Limnology, P.O. Box 1299, 3600 BG

Maarssen, The Netherlands

M. A. Posthumus

Laboratory of Organic Chemistry, Wageningen University,

P.O. Box 8026, $6700 \mathrm{EG}$

Wageningen, The Netherlands

Present address:

J. G. de Boer

Evolutionary Genetics, Centre for Ecological and Evolutionary

Studies, University of Groningen,

P.O. Box 14, 9750 AA Haren, The Netherlands compounds were more strongly induced by multi-species herbivory than expected based on the sum of volatile emissions by each of the herbivores separately, potentially caused by synergistic effects. In contrast, in cucumber, two compounds were suppressed by multi-species herbivory, suggesting the potential for antagonistic effects. We also studied the behavioral responses of the predatory mite Phytoseiulus persimilis, a specialized natural enemy of spider mites. Olfactometer experiments showed that $P$. persimilis preferred volatiles induced by multi-species herbivory to volatiles induced by S. exigua alone or by prey mites alone. We conclude that both lima bean and cucumber plants effectively attract predatory mites upon multi-species herbivory, but the underlying mechanisms appear different between these species.

Keywords Food webs - Indirect defense · Methyl salicylate · Terpenes · Tritrophic interactions · GC-MS .

Signal transduction

\section{Introduction}

Plants can employ arthropod natural enemies and defend themselves against herbivorous insects and mites. Such arthropod natural enemies can have an impact on local herbivore populations and thereby also on plant fitness (Sabelis and van der Meer 1986; Dicke and Sabelis 1989; Pels and Sabelis 1999; van Loon et al. 2000; Fritzsche Hoballah and Turlings 2001; Kessler and Baldwin 2001). Plants can promote the effectiveness of natural enemies by providing alternative food, shelter, or volatiles (Price et al. 1980; Dicke and Sabelis 1988). The emission of herbivoreinduced plant volatiles (HIPV) has been demonstrated in several families (e.g., Dicke et al. 1990a; Turlings et al. 
1990; van den Boom et al. 2004; van Poecke and Dicke 2004). Many parasitoid wasps, predatory mites, and predatory insects use HIPV to locate their prey or hosts (reviewed in Dicke 1999; Dicke and Vet 1999; Sabelis et al. 1999). HIPV are thus thought to have an important influence on the interactions between plants, herbivores, and natural enemies in food webs (Turlings et al. 1995; Dicke and Vet 1999).

To date, studies on the production of HIPV by plants and the responses of natural enemies to these have been carried out mostly for systems with one species of plant, herbivore, and natural enemy. Yet, in the field, most plants are likely to be attacked by several herbivore species, or by herbivores and pathogens, at the same time. It is difficult to predict whether or not changes in HIPV blends upon multi-species herbivory affect attraction of natural enemies, and in what direction. Several studies have attempted to fill this gap by analyzing the chemical composition of volatile blends upon multi-species infestation of plants (Shiojiri et al. 2001; Cardoza et al. 2002; Rodriguez-Saona et al. 2003; Rostás et al. 2006; Moayeri et al. 2007; Rasmann and Turlings 2007; Soler et al. 2007). For instance, Shiojiri et al. (2000) showed that the parasitoid wasp Cotesia plutellae prefers the HIPV blend induced by its host Plutella xylostella to the blend induced by multi-species herbivory by its host and the non-host caterpillar Pieris rapae although volatile blends are similar (Shiojiri et al. 2001). Cotesia glomerata, on the other hand, prefers HIPV blends induced by its host $P$. rapae and non-host caterpillars to blends induced by one caterpillar species feeding alone (Shiojiri et al. 2000; Vos et al. 2001). Cotesia marginiventris and Microplitis rufiventris do not discriminate between the multi-species-induced HIPV blend of plants infested with their host (beet armyworm caterpillars) and a plant pathogen or plants infested with their host alone, although the pathogen significantly suppresses volatile induction (Rostás et al. 2006). Similarly, C. glomerata does not discriminate between mustard plants infested with a root herbivore and its host, or plants infested with its host alone (Soler et al. 2007). The parasitoid C. marginiventris and the entomopathogenic nematode Heterorhabditis megidis exhibited a reduced attraction to volatiles from maize plants that were infested by a non-host in addition to their host, although the non-host was feeding on different tissues from the host, i.e., leaves vs. roots (Rasmann and Turlings 2007).

In this study, we investigated the effects of multi-species herbivory by spider mites (Tetranychus urticae) and beet armyworm caterpillars (Spodoptera exigua) on volatile emissions by two different plant species: lima bean (Phaseolus lunatus) and cucumber (Cucumis sativus), and evaluated the responses of Phytoseiulus persimilis, a specialist natural enemy of spider mites. Both herbivore species are known to induce volatile emission in several plant species, and remarkable differences in HIPV blend composition have been found in lima bean infested by either of these herbivores (e.g., Ozawa et al. 2000; Schmelz et al. 2003; de Boer et al. 2004). We statistically compared volatile emission by multi-species-infested plants with the sum of volatile emissions of plants infested with either herbivore species alone to evaluate the presence of potentially antagonistic or synergistic effects among signal transduction pathways (e.g., Engelberth et al. 2001). Plant responses to multi-species herbivory may depend on feeding modes of the herbivore species involved (Walling 2000), but whether or not plant species differ in these responses is unknown. Therefore, we compared two plant species in their relative responses to multi-species vs. single-species herbivory by using the same two herbivore species to attack both plant species. We also studied the olfactory responses of predatory mites (P. persimilis). In nature, plants are commonly attacked by more than one herbivore. Knowledge on natural enemy responses to volatile blends induced by multi-species vs. single-species herbivory is essential to understand whether differences in volatile composition are relevant in an ecological context.

\section{Methods and Materials}

Plants, Herbivores, and Predators Lima bean plants (Phaseolus lunatus L. cv Sieva) and cucumber plants (C. sativus L. cv Lange Groene Giganten) were grown in a greenhouse at $20-30^{\circ} \mathrm{C}, 50-70 \%$ R.H. and $16 \mathrm{hr}$ of light. Bean plants were used when their primary leaves had unfolded (and were about $8-12 \mathrm{~cm}$ wide), which was 10 $15 \mathrm{~d}$ after sowing. Cucumber plants were used when their first two leaves were about $8-12 \mathrm{~cm}$ wide, which was 15 $35 \mathrm{~d}$ after sowing.

Two-spotted spider mites, Tetranychus urticae Koch (Acari: Tetranychidae), were reared on lima bean plants in a greenhouse under the same conditions as uninfested plants. Eggs of the beet armyworm Spodoptera exigua Hübner (Lepidoptera: Noctuidae) were obtained from a colony reared on artificial diet in the laboratory of Virology, Wageningen University, The Netherlands (Smits et al. 1986). Two to three batches of eggs were placed in a Petri dish together with a lima bean or cucumber leaf in a growth chamber at $23 \pm 1{ }^{\circ} \mathrm{C}$. Larvae were used in experiments within $24 \mathrm{hr}$ of hatching.

A colony of predatory mites, Phytoseiulus persimilis Athias-Henriot (Acari: Phytoseiidae), was kept on spider mite-infested lima bean leaves in a growth chamber at $23 \pm 1{ }^{\circ} \mathrm{C}, 50-70 \%$ R.H. and continuous light. To obtain females of the same age for olfactometer experiments, gravid females were allowed to oviposit on a spider miteinfested bean leaf for $2 \mathrm{~d}$. The female offspring were used 
in experiments $9 \mathrm{~d}$ after initiation of the colonies, i.e., $1-2 \mathrm{~d}$ after their final molt. Before the experiments, females were kept individually in $1.5-\mathrm{ml}$ microcentrifuge tubes with a small droplet of water, for $24 \pm 2 \mathrm{hr}$ at $23 \pm 1{ }^{\circ} \mathrm{C}$.

Plant Treatments Treatments were applied to the two oldest leaves of lima bean or cucumber plants $3 \mathrm{~d}$ before an experiment. Plants were incubated in plastic cages in a climate-controlled chamber at $24 \pm 1{ }^{\circ} \mathrm{C}, 50-70 \%$ R.H. and $16 \mathrm{~L}: 8 \mathrm{D}$, each treatment in a separate cage. We used the following four treatments:

(1) T. urticae: Adult female spider mites were transferred to experimental plants. Twenty mites per leaf were used on lima bean, and 100 mites per leaf were used on cucumber because the same number of spider mites leads to lower attraction of $P$. persimilis to cucumber than to lima bean plants (Dicke et al. 1990b).

(2) S. exigua: Newly hatched S. exigua larvae were placed in clip cages to keep caterpillars on the leaves (clip cages were made of two plastic cylinders, $2.5 \mathrm{~cm}$ diam., and a hairpin; the upper side was covered with gauze and the underside with Parafilm). Two larvae in one clip cage were used per leaf. Clip cages were moved daily to a new position on the same leaf, and any missing larvae were replaced. The clip cages were supported by sticks to prevent the leaves from bending and incurring damage due to the weight of the cages.

(3) Multi-species herbivory: Two newly hatched S. exigua larvae in a clip cage and 20 (lima bean) or 100 (cucumber) T. urticae females were placed on each leaf.

(4) Uninfested: Plants without herbivores were treated in the same way as plants of the other treatments. This treatment was only used for volatile collections of cucumber. We did not analyze volatile emission from uninfested lima bean plants because it is documented that they emit low amounts of volatiles (e.g., de Boer et al. 2004).

When clip cages were used to keep caterpillars in place, T. urticae-infested or uninfested plants were treated in the same way with empty clip cages to avoid any cage effects.

Volatile Collections and Analyses Just before volatile collections, plants were cut above the soil line. Stems of individual plants were wrapped in moist cotton and aluminum foil to prevent them from wilting during volatile collection, which lasted at most $4 \mathrm{hr}$. All clip cages, caterpillars, and their products were removed. Five plants of one treatment were transferred to a 5-1 glass vessel. A viton O-ring and a metal clamp were used to attach the glass lid to the vessel to make it airtight. Purified air (filtered through silica, a molecular sieve, activated charcoal, and $90 \mathrm{mg}$ Tenax) was split into two air streams of about $100 \mathrm{ml} / \mathrm{min}$ each and led into the vessels through teflon tubing. Volatiles from two treatments were collected simultaneously in parallel. The system was purged for 30 min before attaching a collection tube with Tenax (90 $\mathrm{mg}$ for bean samples, $200 \mathrm{mg}$ for cucumber samples) to the air outlet in the lid of the vessel. Bean volatiles were collected for $25 \mathrm{~min}$, cucumber volatiles were collected for $3 \mathrm{hr}$. For lima bean, volatile collection was repeated four times for each treatment, except for uninfested from which volatiles were not sampled. For cucumber, volatile collections were replicated seven times for the uninfested, six times for $T$. urticae, and five times for S. exigua and multi-species herbivory each. One sample of the T. urticae and one of the $S$. exigua treatments of cucumber were lost during the analysis.

Volatiles from lima bean and cucumber were analyzed on two different gas chromatography-mass spectrometry (GCMS) systems.

Lima bean Volatiles were released from Tenax traps with a thermodesorption cold trap setup (Chrompack, Middelburg, The Netherlands) by heating at $250^{\circ} \mathrm{C}$ for $10 \mathrm{~min}$, with a He-flow of $12 \mathrm{ml} / \mathrm{min}$. Desorbed compounds were collected in the cold trap at $-90^{\circ} \mathrm{C}$. Volatiles were injected in splitless mode into the DB5 column $(60 \mathrm{~m} \times 0.25 \mathrm{~mm}$ ID, $0.25 \mu \mathrm{m}$ film thickness) by heating the cold trap to $220^{\circ} \mathrm{C}$. After an initial column temperature of $40^{\circ} \mathrm{C}$ for $4 \mathrm{~min}$, the temperature was raised to $280^{\circ} \mathrm{C}$ at $4^{\circ} \mathrm{C} / \mathrm{min}$. The column was directly coupled to the ion source of a Finnigan MAT 95 mass spectrometer, which was operating in the $70-\mathrm{eV}$ electron impact (EI) ionization mode with scanning from mass 24 to 300 at $0.5 \mathrm{scan} / \mathrm{sec}$. Compounds were identified by comparison of mass spectra with those in the NIST 98 library and in the Wageningen Mass Spectral Database of Natural Products, and by checking the retention indices.

Cucumber Volatiles were released from Tenax traps with a thermodesorption cold trap setup (Markes, UK) by heating at $200^{\circ} \mathrm{C}$ for $10 \mathrm{~min}$, with a He-flow of $30 \mathrm{ml} / \mathrm{min}$. The desorbed volatiles were collected in the cold trap at $-10^{\circ} \mathrm{C}$. Volatiles were injected in splitless mode into the RTX5Silms column (Restec, $30 \mathrm{~m} \times 0.32 \mathrm{~mm}$ ID, $0.33 \mu \mathrm{m}$ film thickness) by heating of the coldtrap to $270^{\circ} \mathrm{C}$. After an initial column temperature of $40^{\circ} \mathrm{C}$ for $2 \mathrm{~min}$, the temperature was raised to $95^{\circ} \mathrm{C}$ at $3^{\circ} \mathrm{C} / \mathrm{min}$, then to $165^{\circ} \mathrm{C}$ at $2^{\circ} \mathrm{C} / \mathrm{min}$, and subsequently to $250^{\circ} \mathrm{C}$ at $15^{\circ} \mathrm{C} / \mathrm{min}$. The column was directly coupled to the ion source of a Finnigan quadrupole mass spectrometer, which was operated in the $70-\mathrm{eV}$ EI ionization mode and scanned from mass 33 to 300 at three scans $/ \mathrm{sec}$. Compounds were identified by comparison of mass spectra with those in the NIST 98 and 
Wiley 7 th edition spectral libraries, and by checking the retention indices.

Analyses Not all compounds were detected in every replicate of every treatment. We therefore added one peak area unit to all measurements for lima bean and 10 units to all measurements for cucumber. These were the lowest recorded non-zero values for bean and cucumber, respectively. This enabled us to plot volatile emission on a $\log$ scale (Figs. 1a and 2a), and to determine ratios among treatments (see below).

For cucumber, we first determined which volatile compounds were induced by herbivory by using KruskalWallis tests per compound. Variation in volatile emission levels of uninfested plants was low, indicating that other types of inducing factors (e.g., general stress) were absent or had only minor effects. Only herbivore-induced volatiles were used in subsequent analyses. We assumed that all
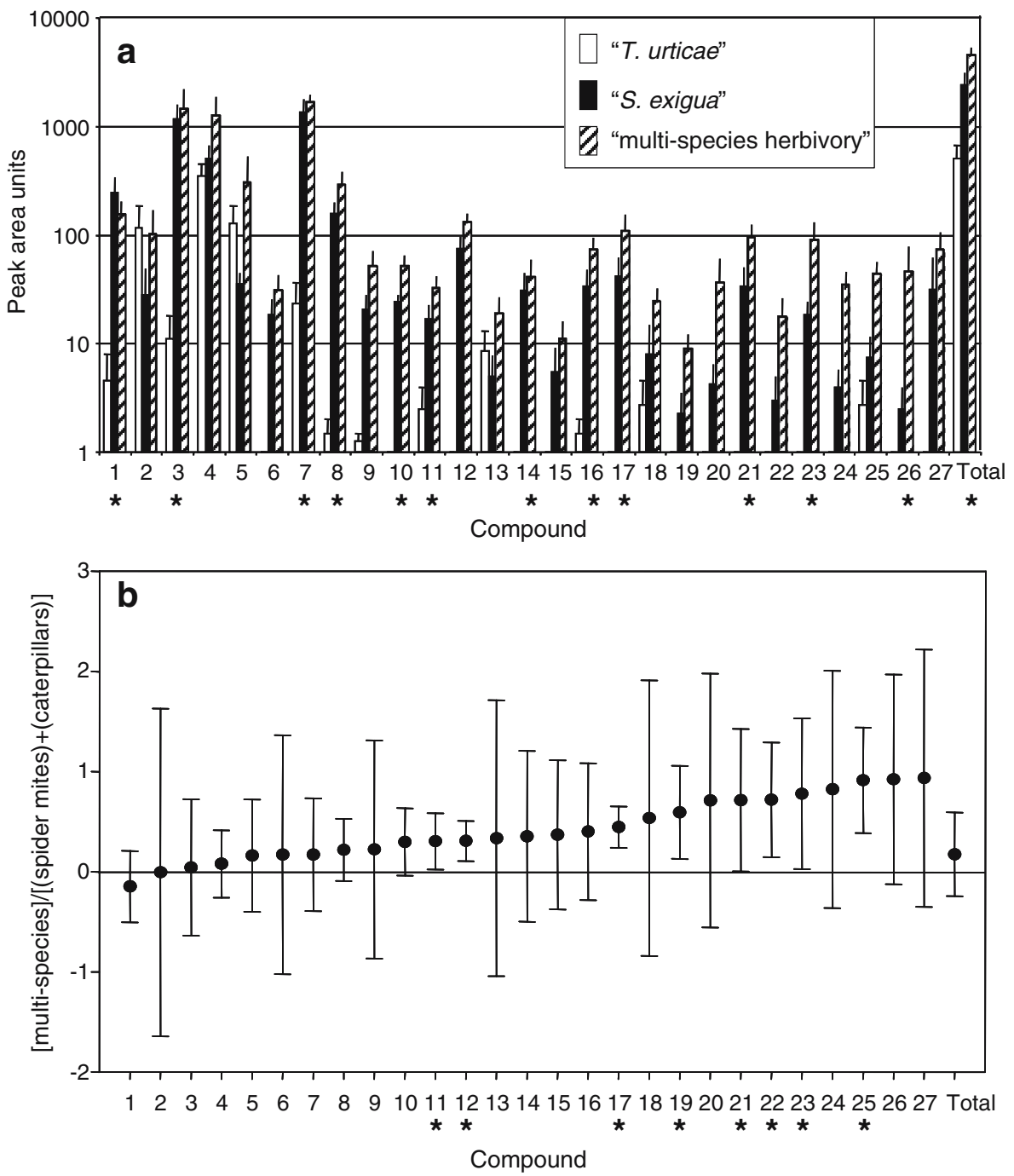

Fig. 1 Effect of multi-species herbivory on volatile emission by lima bean plants. (a) Volatile emission (mean + SE peak area units) upon single herbivory by Tetranychus urticae (20 mites per leaf) (open bars), single herbivory by Spodoptera exigua (two larvae per leaf) (filled bars), or multi-species herbivory (hatched bars). Asterisks indicate compounds that were significantly affected by herbivore treatment $(P<0.05$, Kruskal-Wallis tests). (b) Ratio of emission rates upon multi-species herbivory to the sum per volatile emitted by the two single herbivore treatments (see "Methods and Materials" for detailed explanation). Symbols indicate mean ratio, and error bars indicate upper and lower $95 \%$ confidence limits. Asterisks indicate compounds that were significantly more strongly induced by multispecies herbivory than by the sum of T. urticae and $S$. exigua (lower
$95 \%$ confidence limit larger than 0 ). $N=4$ for all treatments. Compound numbers: (1) (Z)-3-hexen-1-ol, (2) methyl salicylate, (3) (E)- $\beta$-ocimene, (4) (E)-4,8-dimethyl-1,3,7-nonatriene, (5) (3E,7E)4,8,12-trimethyl-1,3,7,11-tridecatetraene, (6) $p$-mentha-1,3,8-triene, (7) (Z)-3-hexen-1-ol acetate, (8) 2-methylbutanal- $O$-methyl oxime, (9) 3-methylbutanal- $O$-methyl oxime, (10) linalool, (11) 1-octen-3-ol, (12) hexyl acetate, (13) limonene, (14) $\beta$-caryophyllene, (15) nonanal, (16) indole, (17) (E)-2-hexen-1-ol acetate, (18) 3-pentanone, (19) 3octanone, (20) 2-methylbutanal nitrile, (21) (Z)- $\beta$-ocimene, (22) rose furan, (23) unknown 95B, 150, (24) 3-methylbutanal nitrile, (25) unknown 91B, 148, (26) unknown 41, 69B, 164, (27) 2-methylpropanal- $O$-methyl oxime 

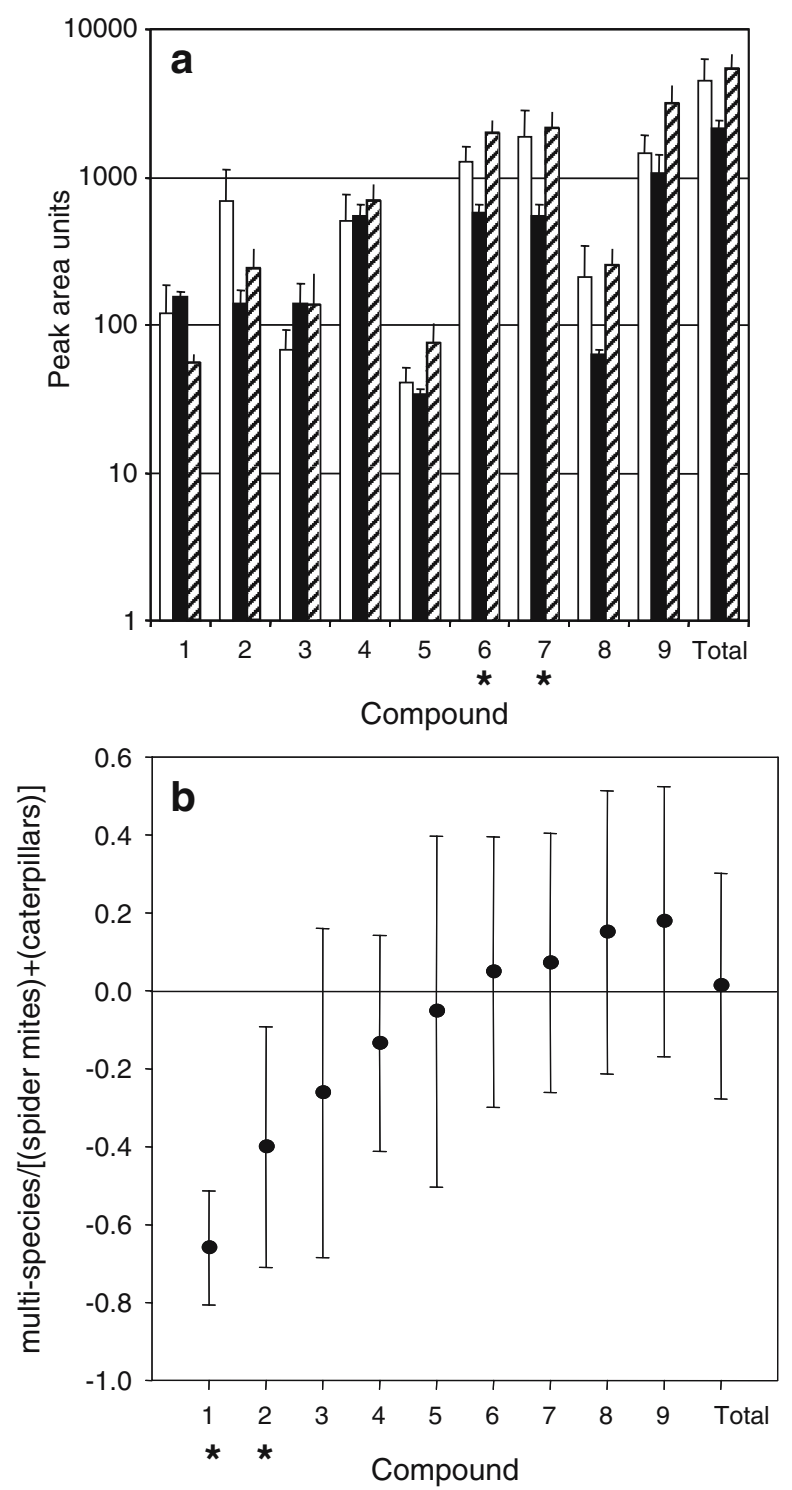

Fig. 2 Effect of multi-species herbivory on volatile emission by cucumber plants. (a) Volatile emission (mean \pm SE peak area units) upon single herbivory by Tetranychus urticae (100 mites per leaf, $N=$ 5, open bars), single herbivory by Spodoptera exigua (2 larvae per leaf, $N=4$, filled bars), or multi-species herbivory $(N=4$, hatched bars). Asterisks indicate compounds that are significantly affected by herbivore treatment $(P<0.05$, Kruskal-Wallis tests). (b) Ratio of emission rates upon multi-species herbivory to the sum per volatile emitted by the two single herbivore treatments (see "Methods and Materials" and for detailed explanation). Symbols indicate mean ratio, and error bars indicate upper and lower 95\% confidence limits. Asterisks indicate compounds that were significantly less induced by multi-species herbivory than by the sum of $T$. urticae and $S$. exigua (upper 95\% confidence limit smaller than 0) $(N=4)$ Compound numbers: (1) (E)-2-hexenal $+(Z)$-3-hexanal, (2) (Z)-3-hexen-1-ol acetate, (3) indole, (4) (E,E)- $\alpha$-farnesene, (5) butyl aldoxime, (6) 3-methylbutanal- $O$-methyl oxime, (7) (E)-4,8-dimethyl-1,3,7-nonatriene, (8) (3E,7E)-4,8,12-trimethyl-1,3,7,11-tridecatetraene, (9) (E)$\beta$-ocimene. Note that peak area units in Fig. 2a cannot be compared with Fig. 1a because measurements were done on a different GC-MS system compounds emitted by infested lima bean plants were induced by herbivores based on previous measurements of volatile emissions by uninfested lima bean plants (e.g., de Boer et al. 2004). Per plant species, we then used KruskalWallis tests to analyze which compounds were differentially induced by the three herbivore treatments (T. urticae, $S$. exigua, or multi-species herbivory) $(\alpha=0.05)$. Note that we did not statistically compare absolute levels of volatile emission across plant species because volatiles were collected for different periods of time, and analyses were run on two different GC-MS systems.

We also tested whether the effect on HIPV of multispecies herbivory was the same as the combined effect of the two herbivores feeding alone, i.e., T. urticae $+S$. exigua. Per replicate, the amount of each volatile compound of the multi-species herbivory treatment was divided by the sum of the amounts emitted by $T$. urticae and $S$. exigua. The ratios were log-transformed before taking their averages. An average ratio of 0 means the effect of multispecies herbivory and the sum of T. urticae and S. exigua is equal. We concluded that a compound was significantly more strongly induced by multi-species herbivory than by the sum of T. urticae and S. exigua if the lower $95 \%$ confidence limit was larger than 0 . Similarly, we concluded a significantly weaker effect of multi-species herbivory if the upper $95 \%$ confidence limit was smaller than 0 (see Figs. 1 and 2). We also used a $\chi^{2}$ test to test whether the number of compounds with a ratio $>0$ and a ratio $<0$ differed from a 50:50 distribution. A 50:50 distribution would be expected if the effect of multi-species herbivory and the sum of T. urticae and $S$. exigua is equal. One replicate of the $T$. urticae treatment in cucumber was excluded from this analysis because we had no corresponding replicates of the $S$. exigua and multi-species herbivory treatments. Note that these tests reflect volatile responses to multi-species herbivory relative to responses to single-species herbivory. This allows us to compare these data between lima bean and cucumber because the data no longer depend on the absolute levels of volatiles detected by the different GC-MS systems.

Y-tube Olfactometer Experiments A closed system Y-tube olfactometer setup was used to test the responses of predatory mites to volatiles induced by multi-species herbivory (Takabayashi and Dicke 1992; de Boer et al. 2004). Odor sources consisted of four leaves of the same treatment that were cut from the plant just before an experiment; the petioles were wrapped in wet cotton wool and aluminum foil. Individual female predators were observed for a maximum of $5 \mathrm{~min}$. A choice was recorded when the finish line, halfway one of the olfactometer arms, was reached within this period. Otherwise, it was recorded as "no-choice". Sixteen to 23 predators were tested per 
experimental day, and each experiment was repeated on 4 different days. Per replicate, new groups of predatory mites and new sets of leaves were used. We tested the responses of predatory mites to volatiles from bean or cucumber plants of the multi-species herbivory treatment vs. volatiles from plants of the T. urticae or S. exigua treatments.

The choices of predatory mites between two odor sources in the Y-tube olfactometer were analyzed with two-sided binomial tests to investigate whether the distribution of the predators differed from 50:50. Predators that did not make a choice were excluded from statistical analyses.

\section{Results}

Volatile Emission by Lima Bean Plants The main compounds emitted by $T$. urticae-infested bean plants were methyl salicylate (MeSA; compound number 2 in Fig. 1a), (3E)-4,8-dimethyl-1,3,7-nonatriene [(E)-DMNT] (4), and $(3 E, 7 E)-4,8,12$-trimethyl-1,3,7,11-tridecatetraene [ $[E, E)$ TMTT] (5). Herbivory by $S$. exigua alone resulted in the emission of similar amounts of (E)-DMNT (4), but amounts of MeSA (2) and (E,E)-TMTT (5) were small. Instead, $S$. exigua-infested bean leaves emitted large amounts of $(E)-\beta$ ocimene (3) and (Z)-3-hexen-1-ol acetate (7). Multi-species herbivory on bean plants induced the same volatile compounds as $S$. exigua feeding alone. Multi-speciesinfested plants emitted larger amounts of all volatile compounds than plants infested with a single herbivore species, except for (Z)-3-hexen-1-ol (1) that was emitted in larger amounts by $S$. exigua-infested plants, and MeSA (2) that was emitted in somewhat larger absolute amounts by $T$. urticae-infested plants.

In lima bean, herbivore treatment had a significant effect on the emission of (Z)-3-hexen-1-ol (1), (E)- $\beta$-ocimene (3), (Z)-3-hexen-1-ol acetate (7), 2-methylbutanal- $O$-methyl oxime (8), linalool (10), 1-octen-3-ol (11), hexyl acetate (12), $\beta$-caryophyllene (14), indole (16), (E)-2-hexen-1-ol acetate (17), (Z)- $\beta$-ocimene (21), and two unknown compounds (23 and 26) (all $P<0.05$, Kruskal-Wallis tests, Fig. 1a). These compounds were all induced in larger amounts by $S$. exigua and/or multi-species herbivory than by $T$. urticae. Total volatile emission was also significantly affected by treatment $(P<0.006$, Kruskal-Wallis test, Fig. 1a).

We compared the ratio of volatiles emitted in the multispecies herbivory treatment to the sum of volatiles emitted by the $T$. urticae and $S$. exigua treatments to determine whether infestation by two herbivore species simultaneously had additive, synergistic or antagonistic effects. Figure $1 b$ shows the average ratio of multi-species herbivory to the sum of T. urticae and S. exigua for lima bean. Eight of
27 compounds were significantly more strongly induced by multi-species herbivory than by the sum of the two herbivore species feeding alone, suggesting synergistic effects (Fig. 1b). These compounds included members of various groups of chemicals, for example, $(E)$-2-hexen-1-ol acetate (17) and ( $Z$ )- $\beta$-ocimene (21). In addition, more compounds had a ratio $>0$ than expected $\left(\chi^{2}=9.80, P=0.002\right)$. None of the compounds was significantly less induced by multispecies herbivory, suggesting the absence of antagonistic effects of T. urticae and S. exigua feeding simultaneously on lima bean.

Volatile Emission by Cucumber Plants Nine compounds were induced by herbivory on cucumber (all $P<0.10$, KruskalWallis tests; data not shown). The main compounds emitted by $T$. urticae-infested cucumber plants were (Z)-3-hexen-1-ol acetate (compound number 2 in Fig. 2a), $(E, E)-\alpha$-farnesene (4), 3-methylbutanal- $O$-methyl oxime (6), (E)-DMNT (7), and (E)- $\beta$-ocimene (9) (Fig. 2a). Similar compounds were emitted in the largest amounts by $S$. exigua-infested cucumber plants. Multi-species herbivory resulted in a volatile blend that consisted of the same compounds as the blends induced upon herbivory by each of the herbivore species alone. The multispecies herbivory treatment resulted in the strongest induction of 3-methylbutanal-O-methyloxime (6) and (E)- $\beta$-ocimene (9), while T. urticae-infested plants emitted the largest amounts of (Z)-3-hexen-1-ol acetate (2). Herbivory by T. urticae alone and multi-species herbivory induced larger amounts of $(E, E)$-TMTT (8) than single herbivory by $S$. exigua alone. No MeSA was detected in any of the cucumber samples.

In cucumber, herbivore treatment had an effect on the emission of 3-methylbutanal-O-methyloxime (6) and $(E)$ DMNT (9) $(P<0.05$, Kruskal-Wallis tests, Fig. 2a). Both compounds were induced in larger amounts by $T$. urticae or multi-species herbivory than by $S$. exigua. Total volatile emission was not affected by herbivore treatment in cucumber $(P>0.10$, Kruskal-Wallis test, Fig. 2a).

Two volatile compounds $[(E)-2$-hexanal/(Z)-3-hexenal (1) and (Z)-3-hexen-1-ol acetate (2)] were induced significantly less by multi-species herbivory than by the sum of $T$. urticae and $S$. exigua (Fig. 2b), suggesting the presence of antagonistic effects of the two herbivore species feeding on cucumber. The seven other compounds were equally induced by multispecies herbivory and the sum of the two herbivores feeding alone, indicating an additive effect of $T$. urticae and S. exigua. The distribution of compounds with a ratio $>0$ or $<0$ did not differ from 50:50 $\left(\chi^{2}=0.06, P=0.81\right)$.

Responses of $\mathrm{P}$. persimilis We tested the preferences of the predatory mite $P$. persimilis for the volatile blends analyzed above in a Y-tube olfactometer. On bean and cucumber plants, predatory mites preferred HIPV induced by multi- 


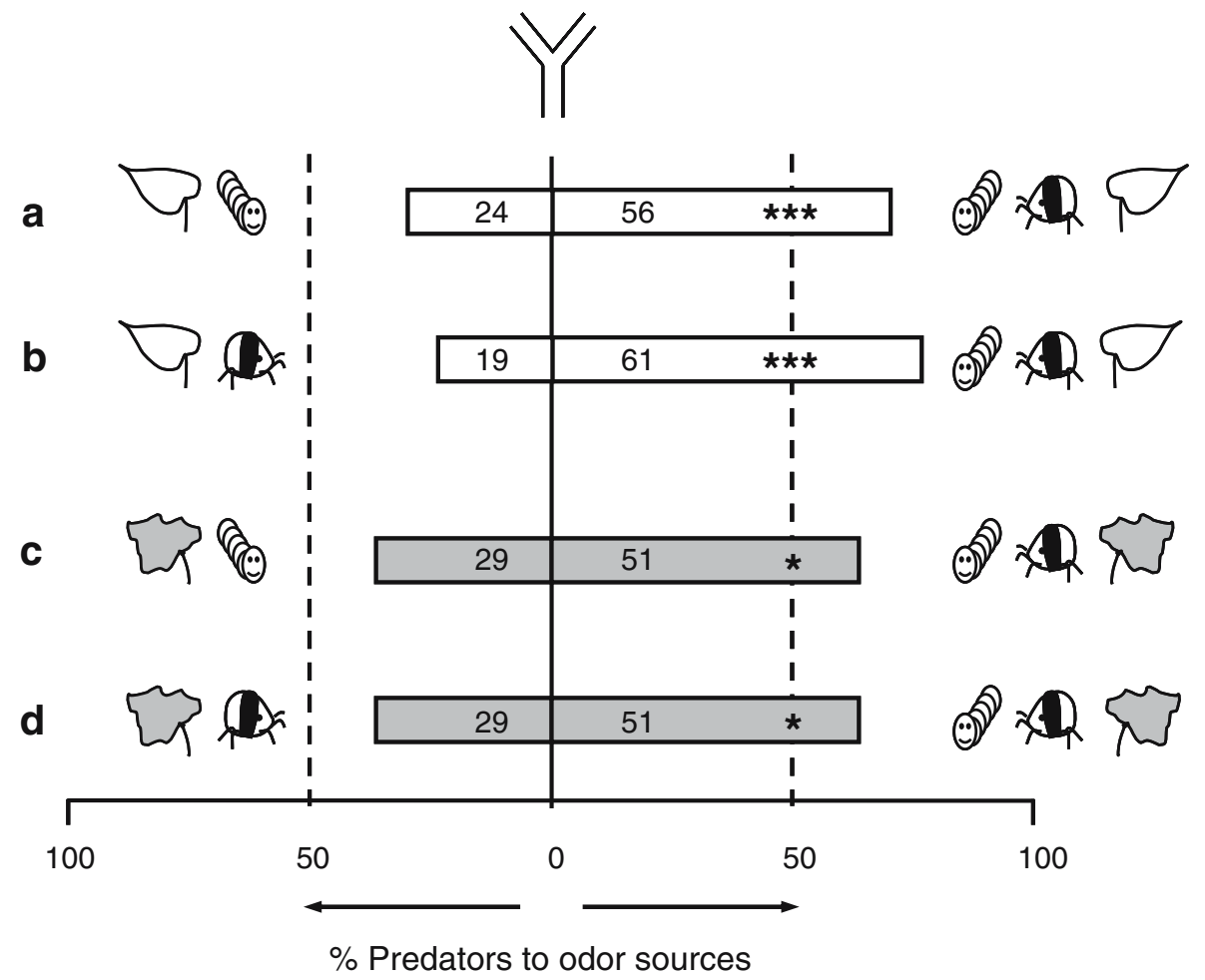

Fig. 3 Responses of $P$. persimilis to volatile blends in the Y-tube olfactometer. Odor sources consisted of four leaves from lima bean (a and b) or cucumber plant (c and d) simultaneously infested by Tetranychus urticae and Spodoptera exigua or infested by one of the herbivore species alone (S. exigua: a and c, T. urticae: b and d). Bars present the overall percentages of predators choosing for each odor source. Numbers in bars are the total numbers of predators responding to each odor source. Choices between odor sources were analyzed with a two-sided binomial test $(* P<0.05 ; * * * P<0.001)$ species herbivory to blends induced by either T. urticae or S. exigua alone (Fig. 3, $P \leq 0.02$, binomial tests).

\section{Discussion}

We investigated volatile emissions by lima bean and cucumber plants upon single and multi-species herbivory by $T$. urticae and $S$. exigua. In lima bean, we found that most compounds were more strongly induced by multispecies herbivory than by the sum of each of the herbivores feeding separately (Fig. 1b). This suggests that the two herbivores feeding together on the same plant have more than an additive effect on volatile emission, potentially indicating that biosynthetic pathways may act synergistically. It is also possible that one or both herbivore species inflicted more damage on multi-species-infested plants than on plants with conspecifics only. However, it is unlikely that spider mite feeding was affected by the presence of $S$. exigua because in a preliminary experiment oviposition rates of mites, which are correlated with food intake rates, were similar on leaf discs with or without $S$. exigua damage (de Boer et al., unpublished). Some studies have observed that feeding by $S$. exigua is enhanced by the presence of aphids or a fungal pathogen (Rodriguez-Saona et al. 2005; Cardoza and Tumlinson 2006), but other studies have found no effect of the presence of whiteflies or a fungus (Rodriguez-Saona et al. 2003; Rostás et al. 2006).

In contrast to bean, we found that in cucumber plants, two volatile compounds were induced less by multi-species herbivory than by the sum of the two herbivores feeding separately, suggesting that their induction was suppressed by simultaneous feeding of T. urticae and S. exigua. Whether this reduction is caused by a negative interaction between the signal transduction pathways induced by spider mites and beet armyworms, or to reduced feeding of one or both herbivore species as a result of feeding on the same plant, remains to be investigated. From our data, it appears that plant species is an important determinant in the way in which herbivore-induced defense pathways interact upon multispecies herbivory.

The plant hormones salicylic acid (SA) and jasmonic acid (JA) play important roles in plant defense pathways and the production of HIPV (e.g., Kessler and Baldwin 2002; van Poecke and Dicke 2004). Interaction between these pathways may occur, for example, when SA inhibits JAdependent defense pathways (e.g., Doares et al. 1995; Niki et al. 1998; Engelberth et al. 2001). To determine whether 
synergistic (lima bean) and antagonistic (cucumber) interactions between biosynthetic defense pathways caused the changes in HIPV blends that we observed, endogenous levels of JA and SA should be determined in future studies. Arimura et al. (2002) showed that both JA and SA levels are significantly elevated in lima bean after $3 \mathrm{~d}$ of spider mite feeding, whereas several Spodoptera species are known to increase JA levels in corn and broad bean (Blechert et al. 1995; Schmelz et al. 2003). We are not aware of analyses of SA levels in response to Spodoptera feeding. No studies of JA and SA levels in plants upon multi-species herbivory are known to us either.

Another important question that needs to be addressed is the effect of herbivore sequence on volatile emission patterns, since in nature two herbivore species may infest an individual plant sequentially. In fact, some herbivore species are known to be attracted to HIPV themselves (e.g., Bolter et al. 1997; Kalberer et al. 2001), but spider mites are not attracted to $S$. exigua-infested lima bean plants (Horiuchi et al. 2003).

To determine whether the differences in volatile profiles upon multi-species herbivory and single-species herbivory are relevant in an ecological context, we investigated the behavior of the predatory mite $P$. persimilis, a specialist natural enemy of spider mites. Our results show that $P$. persimilis prefers volatiles induced by multi-species herbivory by spider mites and the non-prey caterpillar S. exigua to volatiles induced by one of the herbivore species feeding alone (Fig. 3). The strong preference of $P$. persimilis for the volatiles induced by multi-species herbivory may be explained by the relatively large amount of some specific compounds. Of the three major compounds emitted by spider mite-infested lima bean, two, namely $(E, E)$-TMTT and especially $(E)$-DMNT, were induced more strongly by multi-species herbivory, whereas induction of the third, MeSA, was somewhat reduced (Fig. 1a). Of the five major compounds emitted by spider mite-infested cucumber, four were induced more strongly by multi-species herbivory: $(E)$-DMNT, $(E)$ - $\beta$-ocimene, $(E, E)$ $\alpha$-farnesene, and 3-methylbutanal- $O$-methyl oxime, while (Z)-3-hexen-1-ol acetate emission was reduced (Fig. 2a). (E)DMNT, $(E, E)$-TMTT, and $(E)$ - $\beta$-ocimene are known attractants for P. persimilis (Dicke et al. 1990a, de Boer et al. 2004), and (E,E)-TMTT can be used by P. persimilis to discriminate between complex blends of HIPV (de Boer et al. 2004). Similarly, when S. exigua-induced volatiles are compared to the blend induced by multi-species herbivory, all of these compounds are produced in larger amounts in the latter. This suggests that a greater emission of (the sum of) attractive compounds may be the main determinant in attracting the predatory mite $P$. persimilis.

Previous studies on the responses of parasitoid wasps to HIPV also demonstrated a preference for HIPV induced by multi-species herbivory compared to single-species her- bivory (Shiojiri et al. 2000; Vos et al. 2001; Cardoza et al. 2003; Moayeri et al. 2007). Rodriguez-Saona et al. (2005) even showed that naïve Cotesia marginiventris females were only attracted to tomato plants infested with their host $S$. exigua when the same plant was also infested with aphids. The presence of aphids thus made the host-infested plants more detectable. The opposite, i.e., multi-species herbivory-induced volatiles being less attractive than volatiles induced by only the host has been found in two cases: $C$. plutellae wasps preferred volatiles induced by their host, $P$. xylostella, to volatiles induced by their host and the non-host caterpillar P. rapae (Shiojiri et al. 2000), and C. marginiventris as well as the entomopathogenic nematode $H$. megidis were less attracted to volatiles from maize plants that were infested by a non-host in addition to their host (Rasmann and Turlings 2007). In such cases, the presence of non-host or non-prey herbivores may decrease the detectability or reliability of HIPV cues for natural enemies. For example, feeding by a non-prey or non-host herbivore may suppress the induction of volatile compounds used by natural enemies. Multi-species herbivory thus adds a new dimension to the trade-off between reliability and detectability of searching cues used by natural enemies of herbivorous arthropods (Vet and Dicke 1992).

Acknowledgments We thank Wouter Tigges, Leo Koopman, Frans van Aggelen, André Gidding, and Bert Essenstam for rearing plants and spider mites, Els Roode and Magda Usmany from the department of Virology, Wageningen University for providing Spodoptera eggs, and Saskia Burgers for statistical advice. We are grateful to Chris Kulhanek, Remco van Poecke, and Maurice Sabelis for comments on a previous version of this manuscript. JGdB was supported by the Research Council for Earth and Life Sciences of the Netherlands Organization for Scientific Research (NWO-ALW).

Open Access This article is distributed under the terms of the Creative Commons Attribution Noncommercial License which permits any noncommercial use, distribution, and reproduction in any medium, provided the original author(s) and source are credited.

\section{References}

Arimura, G., OzaWa, R., Nishioka, T., Boland, W., Koch, T., KÜHNEMANN, F., and TAKABAYASHI, J. 2002. Herbivore-induced volatiles induce the emission of ethylene in neighboring Lima bean plants. Plant J. 29:87-98.

Blechert, S., Brodschelm, W., Holder, S., KAnNerer, L., Kutcham, T., Mueller, M. J., XiA, Z. Q., and ZenK, M. H. 1995. The octadecanoic pathway: signal molecules for the regulation of secondary patways. Proc. Natl. Acad. Sci. US A 92:4099-4105.

Bolter, C. J., Dicke, M., van LoOn, J. J. A., Visser, J. H., and Posthumus, M. A. 1997. Attraction of Colorado potato beetle to herbivore damaged plants during herbivory and after its termination. J. Chem. Ecol. 23:1003-1023.

CARdozA, Y. J. and Tumlinson, J. H. 2006. Compatible and incompatible Xanthomonas infections differentially affect herbi- 
vore-induced volatile emission by pepper plants. J. Chem. Ecol. 32:1755-1768

CARdozA, Y. J., Alborn, H. T., and Tumlinson, J. H. 2002. In vivo volatile emissions from peanut plants induced by simultaneous fungal infection and insect damage. J. Chem. Ecol. 28:161-174.

CardozA, Y. J., Teal, P. E. A., and Tumlinson, J. H. 2003. Effect of peanut plant fungal infection on oviposition preference by Spodoptera exigua and on host-searching behavior by Cotesia marginiventris. Environ. Entomol. 32:970-976.

De Boer, J. G., Posthumus, M. A., and Dicke, M. 2004. Identification of volatiles that are used in discrimination between plants infested with prey or non-prey herbivores by a predatory mite. J. Chem. Ecol. 30:2215-2230.

DICKE, M. 1999. Evolution of induced indirect defense of plants, pp. 62-88, in R. Tollrian and C. J. Harvell (eds.). The Ecology and Evolution of Inducible Defenses. Princeton University Press, Princeton, New Jersey.

DiCKE M. and SABELIS M. W. 1988. How plants obtain predatory mites as bodyguards. Neth. J. Zool. 38:148-165.

Dicke, M. and SABelis, M. W. 1989. Does it pay plants to advertise for bodyguards? Towards a cost-benefit analysis of induced synomone production, pp. 341-358, in H. Lambers, M. L. Cambridge, H. Konings, and T. L. Pons (eds.). Causes and Consequences of Variation in Growth Rate and Productivity of Higher Plants. SPB Publishing, The Hague, The Netherlands.

DiCKE, M., and VeT, L. E. M. 1999. Plant-carnivore interactions: evolutionary and ecological consequences for plant, herbivore and carnivore, pp. 483-520, in H. Olff, V. K. Brown, and R. H. Drent (eds.). Herbivores: Between Plants and Predators. Blackwell Science, Oxford, U.K.

Dicke, M., van Beek, T. A., Posthumus, M. A., Ben Dom, N., van BoKhoven, H., and DE GROOT, A. E. 1990a. Isolation and identification of volatile kairomone that affects acarine predatorprey interactions. Involvement of host plant in its production. $J$. Chem. Ecol. 16:381-396.

Dicke, M., VAN DER MAas, K. J., TAKabayashi, J., and Vet, L. E. M. 1990b. Learning affects response to volatile allelochemicals by predatory mites. Proc. Exp. Appl. Entomol. 1:31-36.

DoAres, S. H., NARVÁEz-VÁsquez, J., CONCONI, A., and RyAn, C. A. 1995. Salicylic acid inhibits synthesis of proteinase inhibitors in tomato leaves induced by systemin and jasmonic acid. Plant Physiol. 108:1741-1746.

Engelberth, J., Koch, T., SCHÜleR, G., BACHMANN, N., RECHTENBACH, J., and BOLAND, W. 2001. Ion channel-forming alamecithin is a potent elicitor of volatile biosynthesis and tendril coiling. Cross talk between the jasmonate and salicylate signaling in Lima bean. Plant Physiol. 125:369-377.

Fritzsche Hoballah, M. E. and Turlings, T. C. J. 2001. Experimental evidence that plants under caterpillar attack may benefit from attracting parasitoids. Evol. Ecol. Res. 3:553-565.

HoriUchi, J., ARIMURA, G., OzaWA, R., SHIMOdA, T., TAKABAYASHI, J., and NishioKA, T. 2003. A comparison of the responses of Tetranychus urticae (Acari: Tetranychidae) and Phytoseiulus persimilis (Acari: Phytoseiidae) to volatiles emitted from lima bean leaves with different levels of damage made by T. urticae or Spodoptera exigua (Lepidoptera: Noctuidae). Appl. Entomol. Zool. 38:109-116.

Kalberer, N. M., Turlings, T. C. J., and Rahier, M. 2001. Attraction of a leaf beetle (Oreina cacaliae) to damaged host plants. J. Chem. Ecol. 27:647-661.

Kessler, A., and BALDWIN, I. T. 2001. Defensive function of herbivore-induced plant volatile emissions in nature. Science 291:2141-2144

Kessler, A., and Baldwin, I. T. 2002. Plant responses to insect herbivory: The emerging molecular analysis. Annu. Rev. Plant Biol. 53:299-328.
Moayeri, H. R. S., Ashouri, A., Poll, L., and EnKegaArd, A. 2007. Olfactory responses of a predatory mirid to herbivore induced plant volatiles: Multiple herbivory vs. single herbivory. J. Appl. Entomol. 131:326-332.

Niki, T., Mitsuhara, I., SeO, S., OHtsubo, N., and Ohashi, Y. 1998. Antagonistic effect of salicylic acid and jasmonic acid on the expression of pathogenesis-related (PR) protein genes in wounded mature tobacco leaves. Plant Cell Physiol. 39:500-507.

Ozawa, R., Arimura, G., Takabayashi, J., Shimoda, T., and NISHIOKA, T. 2000. Involvement of jasmonate- and salicylaterelated signaling pathways for the production of specific herbivoreinduced volatiles in plants. Plant Cell Physiol. 41:391-398.

PELS, B., and SABELIS, M. W. 1999. Local dynamics, overexploitation and predator dispersal in an acarine predator-prey system. Oikos 86:573-583.

Price, P. W., Bouton, C. E., Gross, P., Mcpheron, B. A., ThOmPSON, J. N., and WeISS, A. E. 1980. Interactions among three trophic levels: Influence of plants on interactions between insect herbivores and natural enemies. Annu. Rev. Ecol. Syst. $11: 41-65$.

RASMANN, S., and TuRLings, T. C. J. 2007. Simultaneous feeding by aboveground and belowground herbivores attenuates plant-mediated attraction of their respective natural enemies. Ecol. Lett. 10:926-936.

Rodriguez-SaOna, C., Crafts-Brandner, S. J., and CaÑas, L. A. 2003. Volatile emissions triggered by multiple herbivore damage: Beet armyworm and whitefly feeding on cotton plants. J. Chem. Ecol. 29:2539-2550.

Rodriguez-Saona, C., Chalmers, J. A., Raj, S., and Thaler, J. S. 2005. Induced plant responses to multiple damagers: differential effects on an herbivore and its parasitoid. Oecologia 143:566-577.

Rostás, M., Ton, J., Mauch-Mani, B., and Turlings, T. C. J. 2006. Fungal infection reduces herbivore-induced plant volatiles of maize but does not affect naïve parasitoids. J. Chem. Ecol. 32:1897-1909.

SABELIS, M. W., and VAN DER MEeR, J. 1986. Local dynamics of the interaction between predatory mites and two-spotted spider mites, pp. 322-344, in J. A. J. Metz and O. Diekmann (eds.). Dynamics of physiologically structured populations. Lecture notes in biomathematics 68. Springer-Verlag, Berlin, Germany.

Sabelis, M. W., Janssen, A., Pallini, A., Venzon, M., Bruin, J., DRUKKER, B., and SCUTAREANU, P. 1999. Behavioral responses of predatory and herbivorous arthropods to induced plant volatiles: From evolutionary ecology to agricultural applications, pp. 269-296, in A. A. Agrawal, S. Tuzun, and E. Bent (eds.). Induced Plant Defenses Against Pathogens and Herbivores. Biochemistry, Ecology and Agriculture. APS Press, St. Paul, Minnesota.

Schmelz, E. A., Alborn, H. T., BAnchio, E., and Tumlinson, J. H. 2003. Quantitative relationships between induced jasmonic acid levels and volatile emission in Zea mays during Spodoptera exigua herbivory. Planta 216:665-673.

ShioJiri, K., TAKabayashi, J., Yano, S., and TaKafuJi, A. 2000. Flight response of parasitoids toward plant-herbivore complexes: A comparative study of two parasitoid-herbivore systems on cabbage plants. Appl. Entomol. Zool. 35:87-92.

ShioJiRI, K., TAKabaYAShi, J., YANO, S., and TAKAFUJi, A. 2001. Infochemically mediated tritrophic interaction webs on cabbage plants. Popul. Ecol. 43:23-29.

SMits, P. H., VAN DE VRIE, M., and VlaK, J. M. 1986. Oviposition of beet armyworm (Lepidoptera: Noctuidae) on greenhouse crops. Environ. Entomol. 15:1189-1191.

Soler R., Harvey, J. A., KAMP, A. F. D., Vet, L. E. M., VAN Der Putten, W. H., van Dam, N. M., Stuefer, J. F., Gols, R., HordiJK, C. A., and BezemER, T. M. 2007. Parasitoids of leaf herbivores repelled by root herbivores. Oikos 116:367-376. 
TAKABAYASHI, J. and DiCKE, M. 1992. Response of predatory mites with different rearing histories to volatiles of uninfested plants. Entomol. Exp. Appl. 64:187-193.

Turlings, T. C. J., Tumlinson, J. H., and Lewis, W. J. 1990. Exploitation of herbivore-induced plant odors by host-seeking parasitic wasps. Science 30:1251-1253.

Turlings, T. C. J., Loughrin, J. H., Mccall P. J., Röse, U. S. R., LEWIS, W. J., and Tumlinson, J. H. 1995. How caterpillardamaged plants protect themselves by attracting parasitic wasps. Proc. Natl. Acad. Sci. U S A 92:4169-4174.

van den Boom, C. E. M., van Beek, T. A., Posthumus, M. A., DE Groot, A., and DiCKE, M. 2004. Qualitative and quantitative variation among volatile profiles induced by Tetranychus urticae feeding on plants from various families. J. Chem. Ecol. 30: 69-89. van Loon, J. J. A., DE Boer, J. G., and Dicke, M. 2000. Parasitoidplant mutualism: Parasitoid attack of herbivore increases plant reproduction. Entomol. Exp. Appl. 97:219-227.

VAN POECKE, R. M. P. and DicKE, M. 2004. Indirect defence of plants against herbivores: Using Arabidopsis thaliana as a model plant. Plant Biol. 6:387-401.

VET, L. E. M. and DICKE, M. 1992. Ecology of infochemical use by natural enemies in a tritrophic context. Annu. Rev. Entomol. $37: 141-172$

Vos, M., Berrocal, S. M., Karamaouna, F., Hemerik, L., and VET, L. E. M. 2001. Plant-mediated indirect effects and the persistence of parasitoid-herbivore communities. Ecol. Lett. $4: 38-45$.

WALLING, L. L. 2000. The myriad plant responses to herbivores. $J$. Plant Growth Regul. 19:195-216. 